\title{
The clinical utility of intraoperative electrocorticography in pediatric epilepsy surgical strategy and planning
}

\author{
*Robert Lesko, MD, ${ }^{1}$ Barbora Benova, MD, PhD, ${ }^{2}$ Petr Jezdik, PhD, ${ }^{3}$ Petr Liby, MD, PhD, \\ Alena Jahodova, MD, PhD, ${ }^{2}$ Martin Kudr, MD, PhD, ${ }^{2}$ Michal Tichy, MD, PhD, ${ }^{1}$ \\ Josef Zamecnik, MD, PhD, ${ }^{4}$ and Pavel Krsek, MD, PhD ${ }^{2}$
}

Departments of ${ }^{1}$ Neurosurgery, ${ }^{2}$ Paediatric Neurology, and ${ }^{4}$ Pathology and Molecular Medicine, Second Faculty of Medicine, Charles University and Motol University Hospital; and ${ }^{3}$ Department of Circuit Theory, Faculty of Electrical Engineering, Czech Technical University of Prague, Czech Republic

\begin{abstract}
OBJECTIVE In this study, the authors aimed to determine 1) whether the use of intraoperative electrocorticography (ECOG) affects outcomes and complication rates of children undergoing resective epilepsy surgery; 2) which patient- and epilepsy-related variables might influence ECoG-based surgical strategy; and 3) what the predictors of epilepsy surgery outcomes are.
\end{abstract}

METHODS Over a period of 12 years, data were collected on pediatric patients who underwent tailored brain resections in the Motol Epilepsy Center. In patients in whom an abnormal ECoG pattern (e.g., spiking, suppression burst, or recruiting rhythm) was not observed beyond presurgically planned resection margins, the authors did not modify the surgical plan (group A). In those with significant abnormal ECoG findings beyond resection margins, the authors either did (group B) or did not (group C) modify the surgical plan, depending on the proximity of the eloquent cortex or potential extent of resection. Using Fisher's exact test and the chi-square test, the 3 groups were compared in relation to epilepsy surgery outcomes and complication rate. Next, multivariate models were constructed to identify variables associated with each of the groups and with epilepsy surgery outcomes.

RESULTS Patients in group $C$ achieved significantly lower rates of seizure freedom compared to groups $A(O R 30.3, p<$ 0.001 ) and $B(O R 35.2, p<0.001)$; groups $A$ and $B$ did not significantly differ $(p=0.78)$. Patients in whom the surgical plan was modified suffered from more frequent complications ( $B$ vs $A+C, O R 3.8, p=0.01$ ), but these were mostly minor (duration < 3 months; $B$ vs $A+C, p=0.008$ ). In all cases, tissue samples from extended resections were positive for the presence of the original pathology. Patients with intended modification of the surgical plan (groups $B+C$ ) suffered more often from daily seizures, had a higher age at first seizure, had intellectual disability, and were regarded as MR-negative $(p<$ 0.001). Unfavorable surgical outcome (Engel class II-IV) was associated with focal cortical dysplasia, incomplete resection based on MRI and/or ECoG findings, negative MRI finding, and inability to modify the surgical plan when indicated.

CONCLUSIONS Intraoperative ECOG serves as a reliable tool to guide resection and may inform the prognosis for seizure freedom in pediatric patients undergoing epilepsy surgery. ECoG-based modification of the surgical plan is associated with a higher rate of minor complications. Children in whom ECoG-based modification of the surgical plan is indicated but not feasible achieve significantly worse surgical outcomes.

https://thejns.org/doi/abs/10.3171/2020.4.PEDS20198

KEYWORDS electrocorticography; epilepsy surgery; drug-resistant epilepsy; epileptogenic zone; interictal epileptiform discharges

$\mathrm{E}$ PILEPSY surgery is an established therapeutic method in adults and children with focal intractable epilepsy, with expected effectiveness approaching $80 \%$ in well-selected patients. ${ }^{1-3}$ The most desirable outcome is to render patients seizure free without new neurological or cognitive deficits. Seizure freedom after surgery depends primarily on the complete removal of the epileptogenic zone (EZ). ${ }^{4,5}$ To achieve complete resection, it is critical to

ABBREVIATIONS ECoG = electrocorticography; EZ = epileptogenic zone; FCD = focal cortical dysplasia; iEEG = invasive EEG; LEAT = low-grade epilepsy-associated brain tumor; $M C D=$ malformation of cortical development; SEEG = stereoelectroencephalography; TSC = tuberous sclerosis complex.

SUBMITTED March 17, 2020. ACCEPTED April 29, 2020

INCLUDE WHEN CITING Published online July 31, 2020; DOI: 10.3171/2020.4.PEDS20198.

${ }^{*}$ R.L. and B.B. contributed equally to this work. 
precisely define borders of the EZ. ${ }^{6}$ Complete resection of the EZ can be verified by intraoperative electrocorticography $(\mathrm{ECoG})$, comparison of preoperative and postoperative MRI, and histopathological assessment of surgical margins. In patients with nonlesional focal epilepsy, this verification is based solely on intracranial EEG study data ${ }^{7}$ and histopathological assessment.

Intraoperative ECoG directly detects epileptiform discharges originating in the epileptogenic cortex during epilepsy surgery. Preresection ECoG, performed immediately before initial resection, aims to determine the extent of the irritative zone. Postresection ECoG aims to identify residual epileptiform discharges remaining after the initial resection. ${ }^{6,8}$ The surgical plan may be modified based on the findings from either preresection or postresection ECoG and allows for the extension of the resection area if epileptiform discharges persist beyond the original surgical margins. Apart from guiding the resection, ECoG findings may serve as a prognostic biomarker of postsurgical seizure freedom, but its predictive value has not yet been clearly defined, especially in patients in whom extension of the resection is not feasible.6.7

Children who undergo epilepsy surgery for focal epilepsy mostly suffer from neocortical extratemporal epilepsy caused by malformations of cortical development (MCDs) or low-grade epilepsy-associated brain tumors (LEATs). ${ }^{9-11}$ The EZ is often widespread and proximal to eloquent cortex. In addition, children often present with inconclusive seizure semiology, and bilateral or generalized scalp EEG patterns, and MRI may fail to detect focal cortical dysplasia (FCD) in infants due to incomplete myelination..$^{12}$ All these factors, in combination with a potential lack of cooperation and a higher risk of neurological complications in young children compared to adults during long-term intracranial EEG monitoring, make identification and precise delineation of the EZ more challenging. ${ }^{13-15}$ These specific features of pediatric epilepsy surgery favor ECoG as the method for delineation of the EZ in this age category.

The ECoG technique, however, possesses certain limitations. The main disadvantages include monitoring of predominantly interictal discharges, limited time and space to get a reliable ECoG signal in the course of ongoing epilepsy surgery, and a high vulnerability to anesthesiologic agents. Experts also disagree about the role of different types of epileptiform patterns and the predictive value of residual discharges on postresection ECoG. ${ }^{8,16}$

Most importantly, data are lacking on how utilization of ECoG in epilepsy surgery planning affects epilepsy surgery outcomes and associated risks, and which groups of patients benefit most from ECoG. The majority of studies on the role of ECoG in epilepsy surgery have focused on specific subgroups of patients and etiologies. To our knowledge, none have addressed the indications and utility of ECoG in the entire spectrum of pediatric patients in everyday epilepsy surgical practice. Therefore, in our study, we analyzed 1) the effect of ECoG on seizure outcome and complication rate, 2) the effect of epilepsy- and patient-related variables on ECoG-guided surgical strategy, and 3) predictors of epilepsy surgery outcomes, including ECoG-guided surgical strategy.

\section{Methods \\ Patient Selection}

We included consecutive pediatric patients who underwent operations between the years 2002 and 2014 at the Motol Epilepsy Center in Prague, Czech Republic. These patients underwent ECoG-guided resective epilepsy surgery for drug-resistant epilepsy, and we had available data on epilepsy course, ECoG findings, epilepsy surgeryrelated complication rates, and seizure outcome, with a minimum 2 years of follow-up. In our practice, ECoG is performed in all patients undergoing resective epilepsy surgery, including selected cases with preceding invasive videoEEG monitoring with subdural (grid or strip) or intracerebral stereoelectroencephalography (SEEG) electrodes, depending on results of invasive videoEEG. We excluded those patients 1) who underwent disconnective surgery (e.g., hemispherotomy), 2) with deep noncortical lesions (e.g., hypothalamic hamartoma), 3) in whom ECoG monitoring failed due to adhesions $(n=4)$ or technical reasons $(\mathrm{n}=1), 4)$ with repeated surgeries for tumor recurrence, and 5) undergoing palliative procedures with no intent for complete resection.

Because the study is observational (retrospective) in nature and no experimental procedures were performed, the approval of the Motol University Hospital ethics committee was not required. Standard informed consent for the epilepsy surgery and for ECoG as a part of the surgical procedure was obtained prior to all procedures from patients or their legal representatives.

\section{Study Design}

We retrospectively analyzed data from patients described above as recorded in the database of the Motol Epilepsy Center. Based on the ECoG findings and resulting potential change in surgical strategy, we divided patients into three groups. Patients in group A had no significant ECoG abnormality (e.g., frequent or continuous spikes, suppression burst, or recruiting rhythm) beyond the margins of presurgically planned resection marked in the neuronavigation system based on assessment of all available diagnostic tests discussed in multidisciplinary meetings (see below) and therefore the original surgical plan was not modified. In patients in group B, the preresection ECoG, postresection ECoG, or both showed an abnormal ECoG pattern beyond the planned resection margins that warranted extension of the resection beyond the original plan, and this modification of the surgical plan was performed. In group $\mathrm{C}$ patients, we were unable to modify the surgical plan despite the presence of an ECoG abnormality, due to the proximity of eloquent cortex or unacceptable extent of resection. Unacceptable resection extent was defined as a situation in which the resection would need to be substantially extended beyond the initial plan, usually beyond margins of the original craniotomy. Extending the craniotomy would prolong the surgery and markedly increase surgical risks.

First, we analyzed whether the three groups differed in their seizure outcome, assessed either by Engel class or simply by the presence/absence of seizures after surgery, and in their complication rate (present, absent) and type (major, minor). Major complications comprised those 
that affected daily life and lasted for a longer period than 3 months, in contrast to minor complications not affecting daily life and lasting less than 3 months. ${ }^{17}$ Next, we searched for patient- and epilepsy-related variables (Table 1) associated specifically with each ECoG study group. Finally, we searched for predictors of seizure outcome in the entire study population. The complete set of analyzed variables can be found in the Supplemental Table.

\section{Presurgical Evaluation and Epilepsy Surgery}

All patients underwent a standard set of presurgical investigations, including long-term videoEEG recording, high-field MRI (1.5 or $3 \mathrm{~T}$ ), functional neuroimaging as indicated (e.g., PET, SPECT, or functional MRI), and neuropsychological testing. In selected cases, invasive EEG (iEEG) studies using either depth SEEG electrodes or subdural strip/grid electrodes were performed. The decision on epilepsy surgery indication and surgical plan was made in multidisciplinary epilepsy surgery group meetings. Based on the results from presurgical evaluation, we always planned the resection extent so as to perform a complete resection. During the surgery itself, we used ECoG to clarify whether the planned extent of the cortical resection was sufficient; based on ECoG findings, the resection might have been extended beyond the originally planned resection margins. In patients in whom extraoperative invasive recording was indicated, $i E E G$ enabled us to localize and approximately delineate the extent of the lesion. However, long-term iEEG, especially SEEG, cannot always precisely define the borders of the lesion and that is why we also used intraoperative ECoG, in addition to extraoperative iEEG.

During the surgery, intraoperative neuronavigation systems were utilized as per the manufacturer's protocol (Brainlab Vector Vision, Brainlab AG, or StealthStation Cranial S7, Medtronic). The resected tissue samples were processed and analyzed in accordance with the published International League Against Epilepsy diagnostic guidelines. ${ }^{18,19}$ Patients who underwent ECoG-guided modification of the surgical plan and subsequent extended resection also had this tissue investigated for the presence of residual pathology (e.g., FCD and tumor).

\section{ECoG Procedure}

Intraoperative ECoG was performed before and after the resective procedure itself (pre- and postresection ECoG, respectively). The ECoG signal was recorded using a combination of several $1 \times 4$ or $1 \times 6$ electrode strips (Integra Lifesciences Inc.) and a 64-channel EEG system (EPAS 32 Schwarzer). The standard settings were as follows: $500-\mathrm{Hz}$ sampling frequency, $1-\mathrm{Hz}$ high-pass, lowpass filter off, $50-\mathrm{Hz}$ notch on, $30 \mathrm{~mm} / \mathrm{sec}$; and $70-\mathrm{Hz}$ lowpass filter in case of artifacts.

Monitoring of the brain surface was usually performed in several positions of strips until accessible cortex was satisfactorily inspected to precisely delineate the EZ margins (preresection $\mathrm{ECoG}$ ) and for the presence of residual epileptogenic tissue (postresection ECoG). In the majority of patients, both pre- and postresection ECoG were performed; the surgical plan could have been modified based on the results either from preresection ECoG or from preand postresection ECoG combined. If the preresection ECoG showed spiking beyond the originally planned resection margins, the resection was extended. After the resection, postresection ECoG was performed and based on its findings the resection was further extended if possible.

Anesthetic agents were utilized in accordance with recommended practices for ECoG procedures; ${ }^{20,21}$ in our center, anesthesia is induced using volatile anesthetic agents and maintained with propofol in the majority of children, in addition to muscle relaxants and analgesics. The standard dose $(8-12 \mathrm{mg} / \mathrm{kg} / \mathrm{hr})$ is continually tapered based on the child's physiological reactions to external stimuli, such as an increase in pulse rate. A full description of the anesthetic protocol was published in a recent study from our center. ${ }^{22}$

ECoG patterns were assessed by an experienced neurophysiologist (P.K., A.J., M.K.) and defined as either nonepileptiform (normal and unspecific abnormity) or epileptiform (sporadic spikes, continuous spikes, burst suppression, and recruiting ictal rhythm) patterns.

\section{Statistical Analysis}

In cases of nominal or ordinal independent variables (presence of group A, B, or C, seizure-free status, and complication status), we performed the contingency tablebased tests such as the chi-square test for $2 \times 2,2 \times 3$, or 3 $\times 3$ tables. In cases of scale variables, we performed nonparametric Kruskal-Wallis tests. If more than two classes were tested, we performed interclass post hoc tests, including Bonferroni's correction. Then, we conducted a univariate regression analysis for continuous observed (independent) variables and selected those with $p$ values $<0.05$ for multivariate analysis. We performed 1-way ANOVA for categorical variables and calculated the median difference and its $95 \%$ confidence interval (CI) using the HodgesLehman estimator. For calculation of effect sizes, $\omega^{2}$ effect size was used for ANOVA tests, $\mathrm{r}^{2}$ effect size for correlation tests and multivariant models, and odds ratio (OR) for categorical data. In the multivariate analysis, we calculated a multiple regression based on the general linear model by stepwise regression algorithm with the variables that reached statistical significance in univariate testing. The beta coefficients with $\mathrm{p}$ values $<0.05$ were considered statistically significant. For the calculations, MATLAB software (version 2017b, MathWorks) and its statistical computing toolbox was used.

\section{Results}

\section{Effect of ECoG on Epilepsy Surgery Outcomes and Complication Rates}

Patients in whom modification of the surgical plan was indicated but could not be performed (group C) had significantly lower rates of seizure freedom compared to patients in whom the surgical plan was modified based on ECoG findings (group B; p < 0.001, OR 35.2, 95\% CI 5.95-208.14) or in whom modification was not indicated (group A; p < 0.001, OR 30.3, 95\% CI 6.04-151.4). Patients in groups $\mathrm{A}$ and $\mathrm{B}$ achieved similar rates of seizure freedom $(\mathrm{p}=0.78)$. 
TABLE 1. Summary of demographic and clinical characteristics

\begin{tabular}{|c|c|c|c|c|}
\hline Variable & Total & Group A & Group B & Group C \\
\hline \multicolumn{5}{|c|}{ Demographic \& clinical characteristics } \\
\hline No. of patients & 141 & 91 & 37 & 13 \\
\hline \multicolumn{5}{|c|}{ Median surgery date (range), yrs } \\
\hline Age at surgery & $11(0.4-18.8)$ & $11(0.8-18.8)$ & $12(0.4-18.7)$ & $9.8(0.9-16.8)$ \\
\hline Seizure onset & $4.5(0.1-16.8)$ & $3.5(0.1-15)$ & $5.9(0.2-16.8)$ & $3(0.1-14.5)$ \\
\hline Epilepsy duration & $4(0.2-18.3)$ & $4.5(0.4-18.3)$ & $3.7(0.2-17.2)$ & $3.5(0.8-12)$ \\
\hline \multicolumn{5}{|l|}{ Sex } \\
\hline Female & $68(50.4)$ & $49(57)$ & $13(35.1)$ & $6(50)$ \\
\hline Male & $67(49.6)$ & $37(43)$ & $24(64.9)$ & $6(50)$ \\
\hline \multicolumn{5}{|l|}{ Seizure frequency } \\
\hline Daily & $76(53.9)$ & $41(45.1)$ & $23(62.2)$ & $12(92.3)$ \\
\hline Weekly & $43(30.5)$ & $32(35.2)$ & $11(29.7)$ & $0(0)$ \\
\hline Monthly or less & $22(15.6)$ & $18(19.8)$ & $3(8.1)$ & $1(7.7)$ \\
\hline \multicolumn{5}{|l|}{ Intellectual disability } \\
\hline No & $106(75.2)$ & $75(82.4)$ & $24(64.9)$ & $7(53.8)$ \\
\hline Yes & $35(24.8)$ & $16(17.6)$ & $13(35.1)$ & $6(46.2)$ \\
\hline \multicolumn{5}{|l|}{ EZ location } \\
\hline Temporal & $68(48.2)$ & $48(52.7)$ & $19(51.4)$ & $1(7.7)$ \\
\hline Extratemporal & $47(33.3)$ & $30(33)$ & $9(24.3)$ & $8(61.5)$ \\
\hline Multilobar/hemispheral & $26(18.4)$ & $13(14.3)$ & $9(24.3)$ & $4(30.8)$ \\
\hline \multicolumn{5}{|l|}{ Lobar extent } \\
\hline Unilobar & $115(81.6)$ & $78(85.7)$ & $28(75.7)$ & $9(69.2)$ \\
\hline Multilobar & $26(18.4)$ & $13(14.3)$ & $9(24.3)$ & $4(30.8)$ \\
\hline \multicolumn{5}{|l|}{ Lobar location } \\
\hline Temporal & $68(59.1)$ & $48(61.5)$ & $19(67.9)$ & $1(11.1)$ \\
\hline Extratemporal & $47(40.9)$ & $30(38.5)$ & $9(32.1)$ & $8(88.9)$ \\
\hline \multicolumn{5}{|l|}{ Pathology } \\
\hline FCD & $39(27.7)$ & $19(20.9)$ & $11(29.7)$ & $9(69.2)$ \\
\hline HS & $31(22)$ & $24(26.4)$ & 7 (18.9) & $0(0)$ \\
\hline Isolated & $13(41.9)$ & $11(45.8)$ & $2(28.6)$ & $0(0)$ \\
\hline w/ FCD & $18(58.1)$ & $13(54.2)$ & $5(71.4)$ & $0(0)$ \\
\hline Tumor & $45(31.9)$ & $33(36.3)$ & $12(32.4)$ & $0(0)$ \\
\hline Isolated & $21(46.7)$ & $16(48.5)$ & $5(41.7)$ & $0(0)$ \\
\hline w/ FCD & $24(53.3)$ & $17(51.5)$ & $7(58.3)$ & $0(0)$ \\
\hline TSC & $11(7.8)$ & $7(7.7)$ & $3(8.1)$ & $1(7.7)$ \\
\hline Others & $15(10.6)$ & $8(8.8)$ & $4(10.8)$ & $3(23.1)$ \\
\hline \multicolumn{5}{|l|}{ Dual pathology } \\
\hline No & $99(70.2)$ & $61(67)$ & $25(67.6)$ & $13(100)$ \\
\hline Yes & $42(29.8)$ & $30(33)$ & $12(32.4)$ & $0(0)$ \\
\hline \multicolumn{5}{|l|}{ FCD } \\
\hline Present & $60(42.6)$ & $42(46.2)$ & $12(32.4)$ & $4(30.8)$ \\
\hline Absent & $81(57.4)$ & $49(53.8)$ & $25(67.6)$ & $9(69.2)$ \\
\hline \multicolumn{5}{|l|}{ Surgery } \\
\hline \multicolumn{5}{|l|}{ Repeated surgery } \\
\hline No & $135(95.7)$ & $86(94.5)$ & $37(100)$ & $12(92.3)$ \\
\hline Yes & $6(4.3)$ & $5(5.5)$ & $0(0)$ & $1(7.7)$ \\
\hline
\end{tabular}


» CONTINUED FROM PAGE 536

TABLE 1. Summary of demographic and clinical characteristics

\begin{tabular}{|c|c|c|c|c|}
\hline Variable & Total & Group A & Group B & Group C \\
\hline \multicolumn{5}{|l|}{ Surgery (continued) } \\
\hline \multicolumn{5}{|l|}{ Long-term iEEG } \\
\hline No & $118(83.7)$ & $78(85.7)$ & $31(83.8)$ & $9(69.2)$ \\
\hline Yes & $23(16.3)$ & $13(14.3)$ & $6(16.2)$ & $4(30.8)$ \\
\hline \multicolumn{5}{|l|}{ Intraop cortical mapping } \\
\hline No & $102(72.3)$ & $63(69.2)$ & $30(81.1)$ & $9(69.2)$ \\
\hline Yes & $39(27.7)$ & $28(30.8)$ & $7(18.9)$ & $4(30.8)$ \\
\hline \multicolumn{5}{|l|}{ Extent of surgery } \\
\hline Less than lobectomy & $53(37.6)$ & $39(42.9)$ & $8(21.6)$ & $6(46.2)$ \\
\hline Lobectomy & $62(44)$ & $39(42.9)$ & $20(54.1)$ & $3(23.1)$ \\
\hline Multilobar resection & $26(18.4)$ & $13(14.3)$ & $9(24.3)$ & $4(30.8)$ \\
\hline \multicolumn{5}{|l|}{ Completeness } \\
\hline \multicolumn{5}{|l|}{ MRI/ECoG } \\
\hline Yes/yes & $105(74.5)$ & $75(82.4)$ & 30 (81.1) & $0(0)$ \\
\hline Yes/no & $10(7.1)$ & $0(0)$ & $2(5.4)$ & $8(61.5)$ \\
\hline No/yes & $17(12.1)$ & $16(17.6)$ & $1(2.7)$ & $0(0)$ \\
\hline No/no & $9(6.4)$ & $0(0)$ & $4(10.8)$ & $5(38.5)$ \\
\hline \multicolumn{5}{|l|}{ MRI \& ECoG } \\
\hline Yes & $105(74.5)$ & $75(82.4)$ & 30 (81.1) & $0(0)$ \\
\hline No & $36(25.5)$ & $16(17.6)$ & 7 (18.9) & $13(100)$ \\
\hline \multicolumn{5}{|l|}{ ECoG } \\
\hline Yes & $122(86.5)$ & $91(100)$ & $31(83.8)$ & $0(0)$ \\
\hline No & $19(13.5)$ & $0(0)$ & $6(16.2)$ & $13(100)$ \\
\hline \multicolumn{5}{|l|}{ MRI } \\
\hline Yes & $115(81.6)$ & $75(82.4)$ & $32(86.5)$ & $8(61.5)$ \\
\hline No & $26(18.4)$ & $16(17.6)$ & $5(13.5)$ & $5(38.5)$ \\
\hline \multicolumn{5}{|l|}{ Outcome } \\
\hline \multicolumn{5}{|l|}{ Seizure control } \\
\hline \multicolumn{5}{|l|}{ Engel classification } \\
\hline Class I & $111(78.7)$ & $77(84.6)$ & $32(86.5)$ & $2(15.4)$ \\
\hline Class II & $5(3.5)$ & $4(4.4)$ & $0(0)$ & $1(7.7)$ \\
\hline Class III & $10(7.1)$ & $5(5.5)$ & $2(5.4)$ & $3(23.1)$ \\
\hline Class IV & $15(10.6)$ & $5(5.5)$ & $3(8.1)$ & $7(53.8)$ \\
\hline \multicolumn{5}{|l|}{ Seizure free } \\
\hline Yes & $111(78.7)$ & $77(84.6)$ & $32(86.5)$ & $2(15.4)$ \\
\hline No & $30(21.3)$ & $14(15.4)$ & $5(13.5)$ & $11(84.6)$ \\
\hline \multicolumn{5}{|l|}{ Complications } \\
\hline No complications & $126(89.4)$ & $85(93.4)$ & $29(78.4)$ & $12(92.3)$ \\
\hline Minor & $11(7.8)$ & $3(3.3)$ & 7 (18.9) & $1(7.7)$ \\
\hline Major & $4(2.8)$ & $3(3.3)$ & $1(2.7)$ & $0(0)$ \\
\hline \multicolumn{5}{|l|}{ Minor } \\
\hline No & $126(89.4)$ & $85(93.4)$ & $29(78.4)$ & $12(92.3)$ \\
\hline Yes & $15(10.6)$ & $6(6.6)$ & $8(21.6)$ & $1(7.7)$ \\
\hline \multicolumn{5}{|l|}{ Major } \\
\hline No & $137(97.2)$ & $88(96.7)$ & $36(97.3)$ & $13(100)$ \\
\hline Yes & $4(2.8)$ & $3(3.3)$ & $1(2.7)$ & $0(0)$ \\
\hline
\end{tabular}


» CONTINUED FROM PAGE 537

TABLE 1. Summary of demographic and clinical characteristics

\begin{tabular}{lcccc}
\hline \multicolumn{1}{c}{ Variable } & Total & Group A & Group B & Group C \\
\hline Outcome (continued) & & & & \\
\hline ECoG findings & & & & $0(0)$ \\
\hline Normal & $2(1.6)$ & $2(2.4)$ & $0(0)$ & $0(0)$ \\
\hline Nonspecific abnormality & $5(4)$ & $5(6)$ & $0(0)$ & $3(33.3)$ \\
\hline Spikes & $74(59.2)$ & $62(74.7)$ & $9(27.3)$ & $6(66.7)$ \\
\hline Continuous spikes & $34(27.2)$ & $13(15.7)$ & $15(45.5)$ & $0(0)$ \\
\hline Burst suppression & $10(8)$ & $1(1.2)$ & $9(27.3)$ & NA \\
\hline Histology of extended resection & & & & NA \\
\hline FCD & $26(70.3)$ & NA & $26(70.3)$ & NA \\
\hline HS & $0(0)$ & NA & $0(0)$ & NA \\
\hline Tumor & $1(2.7)$ & NA & $3(2.7)$ & NA \\
\hline TSC & $3(8.1)$ & NA & $3(8.1)$ & $7(18.9)$ \\
\hline Others & $7(18.9)$ & NA & & \\
\hline
\end{tabular}

HS = hippocampal sclerosis; NA = nonapplicable.

Patients in whom the surgical plan was modified based on ECoG findings (group B) had significantly higher complication rates than patients in whom no modification was indicated (group A; $p=0.013$, OR 3.9, 95\% CI 1.25-12.21) and also compared to those in whom no modification was performed (groups A and C combined; $\mathrm{p}=0.0016$, OR 3.82, 95\% CI 1.28-11.44). Also, patients in group A had significantly lower complication rates than patients in groups $\mathrm{B}$ and $\mathrm{C}$ combined $(\mathrm{p}=0.0356$, OR 3.1, 95\% CI 1.04-9.33); the complication rate did not differ significantly between groups A and C ( $\mathrm{p}=0.88$, OR $1.18,95 \%$ CI 0.13-10.67).

The proportion of major and minor complications ${ }^{17}$ among patients with complications did not differ significantly between groups $\mathrm{A}$ and $\mathrm{B}+\mathrm{C}$ combined $(\mathrm{p}=0.28)$. However, minor complications overall were more prevalent in patients with modification of the surgical plan (group B) compared to those without (groups $\mathrm{A}+\mathrm{C}, \mathrm{p}=$ 0.0075); the prevalence of major complications was not significantly different between the groups $(p=1.0)$.

In all residual tissue samples from extended (modified) resections $(n=37)$, we found histopathological evidence of the original pathology: $\mathrm{FCD}(\mathrm{n}=26)$, tuberous sclerosis complex (TSC; $n=3)$, tumor $(n=1)$, and other pathology $(\mathrm{n}=7)$.

In 23 patients, both extraoperative iEEG and intraoperative ECoG were performed: 13 were group A patients in whom ECoG confirmed the original lesion extent and no modification of surgical plan was warranted, 6 patients belonged in group B and the surgical plan was modified based on ECoG findings, and in 4 patients modification was warranted but impossible to perform.

\section{Patient- and Epilepsy-Related Variables Associated With ECoG Study Groups}

First, using univariate testing we analyzed whether there were any patient- and epilepsy-related variables that would predict whether a patient required modifica- tion of the surgical plan. Next, we selected variables with statistically significant $(\mathrm{p}<0.05)$ associations with group A (Supplemental Table) and constructed a multivariant model using stepwise algorithm. Based on the multivariate model ( $\mathrm{p}$ value of the model $<0.001$ ), we found that the probability that a patient will require modification of the surgical plan (i.e., belongs to groups B or C) increases with increasing patient age, MRI-negative findings, and the presence of intellectual disability and daily seizures. Based on these variables, Fig. 1 illustrates the probability values that a patient will belong to group A $(\mathrm{y}=1)$ versus $\mathrm{B}$ and C combined $(\mathrm{y}=0)$.

\section{Predictors of Epilepsy Surgery Outcomes}

For the analysis of the predictors of epilepsy surgery outcome after 2 years of follow-up, we utilized the same methodology as described above. After we selected variables significantly $(\mathrm{p}<0.05)$ associated with seizure-free outcome in univariate testing, we constructed a multivariate model, retaining the variable "presence in the group $\mathrm{A} / \mathrm{B} / \mathrm{C}$ " ( $\mathrm{p}$ value of the model $<0.001$ ). The seizure-free outcome was determined by histopathological findings, the type of FCD, whether the patient had an MRI-negative finding, and ECoG group. Patients who had complete resection based on postsurgical MRI and ECoG findings with LEAT who belonged in group B had the highest chances for seizure freedom. The probability of seizurefree outcome depending on the studied variables is depicted in Fig. 2 ( $\mathrm{y}=1$ corresponds to $100 \%$ vs $\mathrm{y}=0$ corresponds to $0 \%$ chance of seizure freedom).

\section{Discussion}

In this study, we described how ECoG-guided modification of surgical strategy affects seizure outcomes and complication rates in children undergoing ECoG-guided epilepsy surgery. We also analyzed variables associated with three distinct ECoG-guided surgical strategies and predictors of epilepsy surgery outcome. 

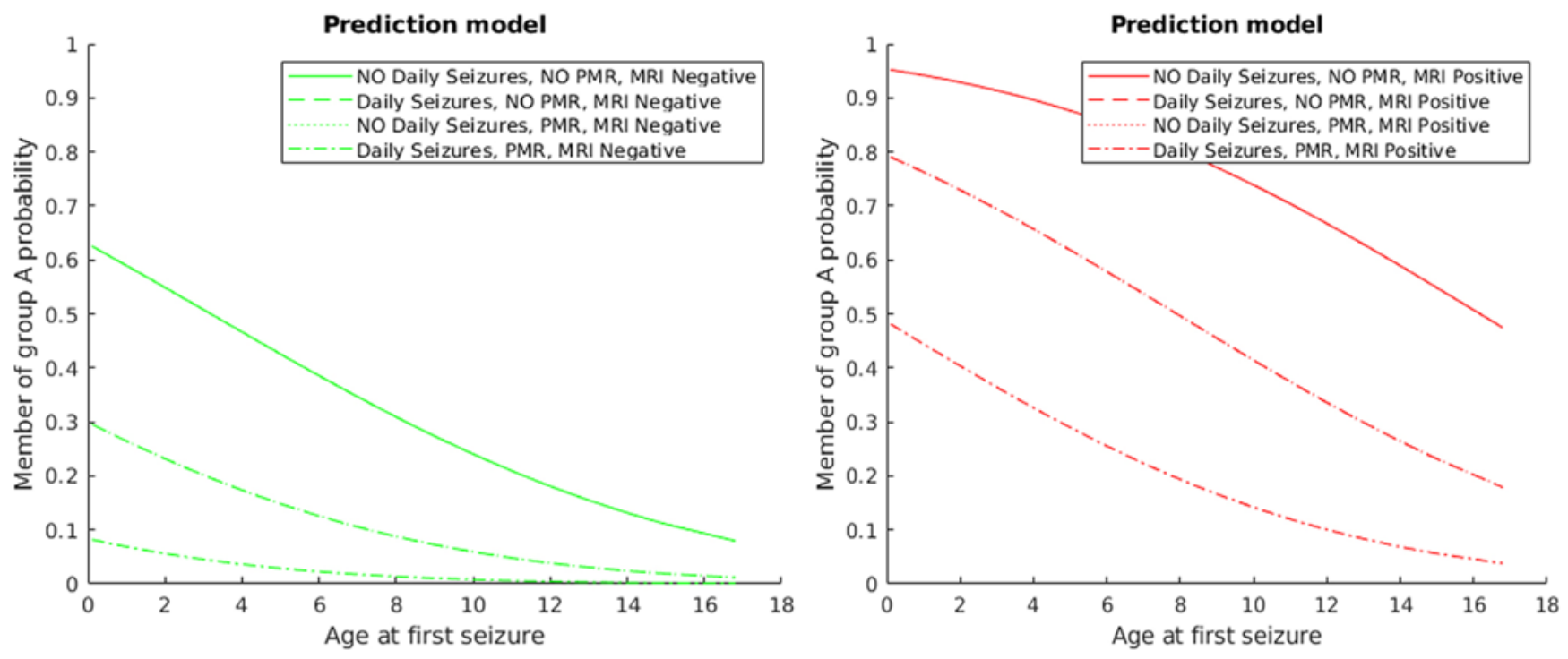

FIG. 1. Prediction model for probability of indicated modification of the surgical plan. The $y$-axis illustrates the probability that a patient would belong to group $A$ (no indication for modification of surgical plan) versus groups $B$ and $C$ combined (indication for modification of the surgical plan); $y=1$ signifies a $100 \%$ probability that a patient belongs to group $A$ and $0 \%$ probability that he or she belongs to groups B and C combined. Left: Prediction model for patients with MRI-negative findings. Right: Prediction model for patients with distinct lesions on MRI (MRI-positive). PMR = psychomotor retardation. Figure is available in color online only.

We established that patients who underwent ECoGguided modification of the surgical plan (extension of the planned resection; group B) achieved significantly higher rates of seizure freedom than those in whom the modification was not feasible (e.g., due to overlapping EZ with eloquent cortical areas and other surgical reasons; group C). The complication rate was higher overall in patients who required modification of the surgical plan, but these complications were transitory and did not affect daily life. Patients who warranted modification of the surgical plan were characterized by increasing patient age, MRInegative findings, intellectual disability, and daily seizures. Histopathological diagnosis, including FCD type, normal MRI findings, and ECoG group, determined the probability of postsurgical seizure freedom. Our results thus significantly contribute to the ongoing discussion of the practical value of ECoG in tailoring resections in children with focal intractable epilepsy.
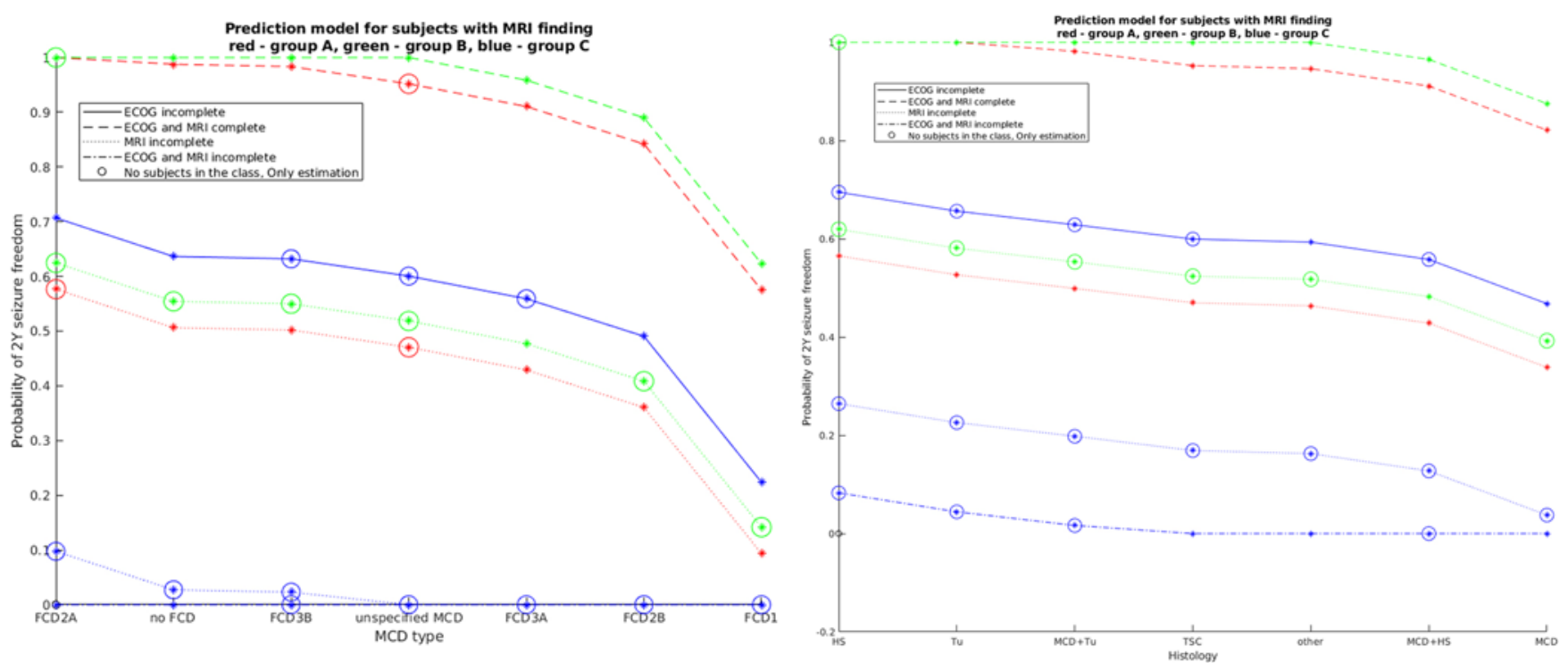

FIG. 2. Prediction model for completeness of resections according to ECoG and MRI findings in individual histopathological groups. The $y$-axis illustrates the probability of a seizure-free outcome after 2 years of follow-up. Left: Prediction model for patients with MRI-negative findings. Right: Prediction model for patients with distinct lesion on MRI (MRI-positive). Tu = tumor. Figure is available in color online only. 
The effect of ECoG on epilepsy surgery outcomes remains controversial. Some studies have shown that patients with ECoG-guided resections achieve favorable seizure outcome, ${ }^{23-26}$ while others have disputed it. ${ }^{27-29}$ The association of epilepsy surgery outcomes and completeness of resection assessed by ECoG was observed in some studies $^{16,26,30}$ and refuted in others. ${ }^{31-34}$ Our study shows that, indeed, patients with epileptiform discharges beyond the original resection margins in whom resection cannot be extended (e.g., due to the presence of eloquent cortex) achieve significantly worse surgical outcomes compared to those in whom ECoG confirmed the initial surgical plan. Also, histopathological assessment of tissue samples from extended resection margins showed the presence of the original pathology (e.g., FCD or LEAT) beyond the planned resection margins. These results provide evidence for the prognostic value and diagnostic utility of ECoG findings; however, the effect of the ECoG procedure itself on epilepsy surgery outcomes would warrant a randomized trial.

In the next step, we analyzed whether ECoG-guided modification of the surgical plan increases complication rates. ECoG is generally considered a safe technique; possible complications may be related to craniotomy and epilepsy surgery, but can rarely be attributed to the $\mathrm{ECoG}$ procedure itself. ${ }^{6}$ However, additional tissue resection may increase the risk of further neurological deficits and thus represents the main ECoG-related risk. ${ }^{34,35}$ In our study, ECoG-guided modifications carried a higher risk of complications; patients in group B had higher complication rates compared to those in groups $\mathrm{A}$, or $\mathrm{A}$ and $\mathrm{C}$ combined, and the complication rates between groups $\mathrm{A}$ and $\mathrm{C}$ were not significantly different; therefore, group $\mathrm{C}$ did not appear to contribute to the overall complication rate. Patients who underwent ECoG-based modification of the surgical plan suffered more frequently from minor complications, but the rate of major complications (i.e., those lasting longer than 3 months and/or affecting daily life ${ }^{17}$ ) was similar between the studied groups. We therefore presume that extension of resection, especially in proximity to eloquent cortical areas, may lead to transient neurological deficits, e.g., transient motor or speech deficit due to perifocal edema. However, these neurological deficits tend to recede. Major complications seem to be unrelated to the ECoG-based modification of surgical plans because they occur with similar frequency in the studied groups.

In light of potential (albeit transitory) complications associated with ECoG, it is important to know which patients benefit most from ECoG. Our findings show that ECoGbased modification was indicated more often in patients with daily seizures, those with intellectual disability, those with MRI-negative findings, and older children. This may be attributed to the fact that in older children we are aiming for more limited resections due to a less extensive $\mathrm{EZ}^{10}$ and lack of brain plasticity, compared to infants and small children. Frequent seizures, intellectual disability, and MRI-negative findings are all typical features of FCD, and FCD is the most common etiology in pediatric epilepsy surgical series. ${ }^{2,10}$ Multiple authors have provided evidence for the benefit of ECoG in guiding surgical strategy and also for predicting outcome in children with FCD. ${ }^{36-38}$
The value of ECoG remains disputable in patients with tumors and TSC. Most studies have shown that gross-total resection of the tumor is the main positive predictive factor of postsurgical seizure freedom, without significant effect of ECoG; ${ }^{39}$ other studies, however, suggested a diagnostic role of ECoG in detecting specific patterns of FCD associated with LEAT. ${ }^{40}$ The role of ECoG in children with TSC and other multilesional pathologies remains to be elucidated, but ECoG reportedly contributes to epilepsy surgery planning in TSC patients. ${ }^{41}$

In our center, we tend to perform invasive extraoperative EEG (SEEG/grids/strips) less frequently (in approximately $16 \%$ of patients ${ }^{42}$ than what is the practice in other European centers $(29.5 \%){ }^{43}$ In recent years, we have successfully established the methods of intraoperative electrophysiological investigations: the use of intraoperative ECoG, presented in this study, and of intraoperative electrical stimulation mapping of motor functions, as published previously. ${ }^{22}$ The combined use of these methods has enabled us to reliably extend resection margins when necessary and to safely perform resections in the proximity of eloquent cortical areas. In properly selected patients, therefore, this can be performed even without the need for long-term extraoperative iEEG. To summarize, in our experience long-term extraoperative iEEG and intraoperative ECoG are complementary electrophysiological methods that can both increase the chances for complete resections.

Our study has multiple limitations; the patients were not randomized and there was no control group. Given the fact that ECoG currently represents the standard of care and has been implemented in our center for many years in patients undergoing epilepsy surgery, randomization would not have been possible. The patients included in the study were heterogenous, including lesional and nonlesional cases, those who underwent invasive extraoperative EEG studies, and those who did not. It would have been ideal to study the groups separately, but the small number of patients in each of the groups would have precluded reasonable statistical analysis.

Despite the limitations, our results show that patients with ECoG-based modification of the surgical plan achieve more favorable outcomes than those in whom modification was either not feasible or not indicated. Several factors were associated with unfavorable surgical outcome in our series, including normal/nonspecific MRI findings and incomplete resection (based on both MRI and ECoG). Our observations are generally consistent with previously published outcome predictors. ${ }^{44,45}$ In addition, our findings provide evidence that the use of intraoperative ECoG may serve as one of the reliable predictors of postsurgical seizure outcome across the entire pediatric epilepsy surgery population. We thus suggest that carefully interpreted ECoG findings could assist in postsurgical patient management, including the process of antiepileptic drug withdrawal.

\section{Conclusions}

In the present study, we established that patients who underwent ECoG-guided modification of the surgical plan 
(extension of the planned resection) achieved significantly higher rates of seizure freedom than those in whom the modification was not feasible (e.g., due to the presence of eloquent cortical area). The modification carried a higher risk of minor complications, but the rate of major complications was similar between the studied groups and was therefore presumably unrelated to the ECoG procedure. Patients who warranted modification of the surgical plan were characterized by increasing patient age, normal MRI findings, intellectual disability, and daily seizures. Histopathological diagnosis, including FCD type, MRI-negative findings, and ECoG group, determined the probability of postsurgical seizure freedom. Patients in whom modification was not feasible achieved significantly less favorable seizure outcomes. Unfavorable surgical outcome was also associated with normal MRI findings and incomplete resection (based on MRI and ECoG). Intraoperative ECoG therefore represents a reliable tool that may contribute to a more precise delineation of the margins of the resection area, in addition to established noninvasive and invasive methods of presurgical evaluation. In selected patient groups, ECoG findings can also provide additional information on the chances for postsurgical seizure freedom.

\section{Acknowledgments}

We would like to thank our patients and their families for their participation in the study. Also, we would like to thank the clinical teams at the Department of Neurosurgery and Department of Paediatric Neurology, Second Faculty of Medicine, Charles University and Motol University Hospital, Prague, Czech Republic. The work was supported by a grant to $\mathrm{MH}-\mathrm{CZ}$ DRO University Hospital Motol, Prague, Czech Republic (no. 00064203-6005), and by the Ministry of Health of the Czech Republic (grant no. NV19-04-00369).

\section{References}

1. Ryvlin P, Cross JH, Rheims S. Epilepsy surgery in children and adults. Lancet Neurol. 2014;13(11):1114-1126.

2. Blumcke I, Spreafico R, Haaker G, et al. Histopathological findings in brain tissue obtained during epilepsy surgery. $N$ Engl J Med. 2017;377(17):1648-1656.

3. Dwivedi R, Ramanujam B, Chandra PS, et al. Surgery for drug-resistant epilepsy in children. $N$ Engl J Med. 2017; 377(17):1639-1647.

4. Rosenow F, Lüders H. Presurgical evaluation of epilepsy. Brain. 2001;124(pt 9):1683-1700.

5. Krsek P, Maton B, Jayakar P, et al. Incomplete resection of focal cortical dysplasia is the main predictor of poor postsurgical outcome. Neurology. 2009;72(3):217-223.

6. Fernández IS, Loddenkemper T. Electrocorticography for seizure foci mapping in epilepsy surgery. J Clin Neurophysiol. 2013;30(6):554-570.

7. Chang EF, Wang DD, Barkovich AJ, Tihan T. Predictors of seizure freedom after surgery for malformations of cortical development. Ann Neurol. 2011;70(1):151-162.

8. Greiner HM, Horn PS, Tenney JR, et al. Should spikes on post-resection ECoG guide pediatric epilepsy surgery? Epilepsy Res. 2016;122:73-78.

9. Duchowny M, Jayakar P, Resnick T, et al. Epilepsy surgery in the first three years of life. Epilepsia. 1998;39(7):737-743.

10. Harvey AS, Cross JH, Shinnar S, Mathern GW. Defining the spectrum of international practice in pediatric epilepsy surgery patients. Epilepsia. 2008;49(1):146-155. Published correction in Epilepsia. 2013;54(6):1140
11. Bansal S, Kim AJ, Berg AT, et al. Seizure outcomes in children following electrocorticography-guided single-stage surgical resection. Pediatr Neurol. 2017;71:35-42.

12. Eltze CM, Chong WK, Bhate S, et al. Taylor-type focal cortical dysplasia in infants: some MRI lesions almost disappear with maturation of myelination. Epilepsia. 2005;46(12): 1988-1992.

13. Hemb M, Velasco TR, Parnes MS, et al. Improved outcomes in pediatric epilepsy surgery: the UCLA experience, 19862008. Neurology. 2010;74(22):1768-1775.

14. Hader WJ, Tellez-Zenteno J, Metcalfe A, et al. Complications of epilepsy surgery: a systematic review of focal surgical resections and invasive EEG monitoring. Epilepsia. 2013;54(5): 840-847.

15. Vendrame M, Kaleyias J, Loddenkemper T, et al. Electroencephalogram monitoring during intracranial surgery for moyamoya disease. Pediatr Neurol. 2011;44(6):427-432.

16. Greiner HM, Horn PS, Tenney JR, et al. Preresection intraoperative electrocorticography (ECoG) abnormalities predict seizure-onset zone and outcome in pediatric epilepsy surgery. Epilepsia. 2016;57(4):582-589.

17. Bjellvi J, Flink R, Rydenhag B, Malmgren K. Complications of epilepsy surgery in Sweden 1996-2010: a prospective, population-based study. J Neurosurg. 2015;122(3):519-525.

18. Blümcke I, Thom M, Aronica E, et al. The clinicopathologic spectrum of focal cortical dysplasias: a consensus classification proposed by an ad hoc Task Force of the ILAE Diagnostic Methods Commission. Epilepsia. 2011;52(1):158-174.

19. Blümcke I, Mühlebner A. Neuropathological work-up of focal cortical dysplasias using the new ILAE consensus classification system - practical guideline article invited by the EuroCNS Research Committee. Clin Neuropathol. 2011;30(4): 164-177.

20. Shetty A, Pardeshi S, Shah VM, Kulkarni A. Anesthesia considerations in epilepsy surgery. Int J Surg. 2016;36(pt B): 454-459.

21. Chidambaran V, Costandi A, D’Mello A. Propofol: a review of its role in pediatric anesthesia and sedation. CNS Drugs. 2015;29(7):543-563.

22. Jahodová A, Beňová B, Kudr M, et al. A novel effective paradigm of intraoperative electrical stimulation mapping in children. J Neurosurg Pediatr. Published online April 17, 2020. doi:10.3171/2020.2.PEDS19451

23. Sugano H, Shimizu H, Sunaga S. Efficacy of intraoperative electrocorticography for assessing seizure outcomes in intractable epilepsy patients with temporal-lobe-mass lesions. Seizure. 2007;16(2):120-127.

24. Stefan H, Nimsky C, Scheler G, et al. Periventricular nodular heterotopia: a challenge for epilepsy surgery. Seizure. 2007; 16(1):81-86.

25. Tripathi M, Garg A, Gaikwad S, et al. Intra-operative electrocorticography in lesional epilepsy. Epilepsy Res. 2010;89(1): 133-141.

26. Gelinas JN, Battison AW, Smith S, et al. Electrocorticography and seizure outcomes in children with lesional epilepsy. Childs Nerv Syst. 2011;27(3):381-390.

27. Cascino GD, Trenerry MR, Jack CR Jr, et al. Electrocorticography and temporal lobe epilepsy: relationship to quantitative MRI and operative outcome. Epilepsia. 1995;36(7):692-696.

28. Kanazawa O, Blume WT, Girvin JP. Significance of spikes at temporal lobe electrocorticography. Epilepsia. 1996;37(1): $50-55$.

29. Tran TA, Spencer SS, Javidan M, et al. Significance of spikes recorded on intraoperative electrocorticography in patients with brain tumor and epilepsy. Epilepsia. 1997;38(10):11321139 .

30. Burkholder DB, Sulc V, Hoffman EM, et al. Interictal scalp electroencephalography and intraoperative electrocorticography in magnetic resonance imaging-negative temporal lobe epilepsy surgery. JAMA Neurol. 2014;71(6):702-709. 
31. Schramm J. Temporal lobe epilepsy surgery and the quest for optimal extent of resection: a review. Epilepsia. 2008;49(8): $1296-1307$.

32. San-juan D, Tapia CA, González-Aragón MF, et al. The prognostic role of electrocorticography in tailored temporal lobe surgery. Seizure. 2011;20(7):564-569. Published correction in Seizure. 2012;21(5):410.

33. Wray CD, McDaniel SS, Saneto RP, et al. Is postresective intraoperative electrocorticography predictive of seizure outcomes in children? J Neurosurg Pediatr. 2012;9(5):546-551.

34. Wong CH, Bleasel A, Wen L, et al. Relationship between preoperative hypometabolism and surgical outcome in neocortical epilepsy surgery. Epilepsia. 2012;53(8):1333-1340.

35. Tanriverdi T, Kemerdere R, Baran O, et al. Long-term surgical and seizure outcomes of frontal low-grade gliomas. Int $J$ Surg. 2016;33(pt A):60-64.

36. Knerlich-Lukoschus F, Connolly MB, Hendson G, et al. Clinical, imaging, and immunohistochemical characteristics of focal cortical dysplasia Type II extratemporal epilepsies in children: analyses of an institutional case series. J Neurosurg Pediatr. 2017;19(2):182-195.

37. Gröppel G, Dorfer C, Samueli S, et al. Single stage epilepsy surgery in children and adolescents with focal cortical dysplasia type II-prognostic value of the intraoperative electrocorticogram. Clin Neurophysiol. 2019;130(1):20-24.

38. van 't Klooster MA, van Klink NEC, Zweiphenning WJEM, et al. Tailoring epilepsy surgery with fast ripples in the intraoperative electrocorticogram. Ann Neurol. 2017;81(5): 664-676.

39. Englot DJ, Berger MS, Barbaro NM, Chang EF. Predictors of seizure freedom after resection of supratentorial low-grade gliomas. A review. J Neurosurg. 2011;115(2):240-244.

40. Ferrier CH, Aronica E, Leijten FSS, et al. Electrocorticographic discharge patterns in glioneuronal tumors and focal cortical dysplasia. Epilepsia. 2006;47(9):1477-1486.

41. Arya R, Tenney JR, Horn PS, et al. Long-term outcomes of resective epilepsy surgery after invasive presurgical evaluation in children with tuberous sclerosis complex and bilateral multiple lesions. J Neurosurg Pediatr. 2015;15(1):26-33.

42. Belohlavkova A, Jezdik P, Jahodova A, et al. Evolution of pediatric epilepsy surgery program over 2000-2017: improvement of care? Eur J Paediatr Neurol. 2019;23(3):456-465.
43. Baud MO, Perneger T, Rácz A, et al. European trends in epilepsy surgery. Neurology. 2018;91(2):e96-e106.

44. Ryvlin P, Rheims S. Predicting epilepsy surgery outcome. Curr Opin Neurol. 2016;29(2):182-188.

45. Malmgren K, Edelvik A. Long-term outcomes of surgical treatment for epilepsy in adults with regard to seizures, antiepileptic drug treatment and employment. Seizure. 2017;44: 217-224.

\section{Disclosures}

The authors report no conflict of interest concerning the materials or methods used in this study or the findings specified in this paper.

\section{Author Contributions}

Conception and design: all authors. Acquisition of data: Krsek, Lesko, Benova, Liby, Jahodova, Kudr, Tichy, Zamecnik. Analysis and interpretation of data: all authors. Drafting the article: Krsek, Lesko, Benova. Critically revising the article: Krsek, Lesko, Benova, Jezdik, Liby, Jahodova, Kudr. Reviewed submitted version of manuscript: all authors. Approved the final version of the manuscript on behalf of all authors: Krsek. Statistical analysis: Benova, Jezdik. Study supervision: Krsek, Tichy, Zamecnik.

\section{Supplemental Information Online-Only Content}

Supplemental material is available with the online version of the article.

Supplemental Table. https://thejns.org/doi/suppl/10.3171/2020. 4.PEDS20198.

\section{Correspondence}

Pavel Krsek: Charles University in Prague, Second Faculty of Medicine, Motol University Hospital, Prague, Czech Republic. pavel.krsek@post.cz. 